\title{
DURUM WHEAT ROOTS ADAPT TO SALINITY REMODELLING THE CELLULAR CONTENT OF NITROGEN METABOLITES AND SUCROSE
}

\author{
Annunziata Maria Grazia ${ }^{1}$, Ciarmiello Loredana $F^{2}$, Woodrow Pasqualina ${ }^{2}$, \\ Maximova Eugenia ${ }^{1}$, Fuggi Amodio ${ }^{2}$ and Carillo Petronia ${ }^{2}$ \\ 1 Department of Metabolic Networks, Max Planck Institute of Molecular Plant Physiology, Potsdam, \\ Germany, 2 Dipartimento di Scienze e Tecnologie Ambientali, Biologiche e Farmaceutiche, Università degli \\ Studi della Campania "Luigi Vanvitelli", Caserta, Italy \\ e-mail: petronia.carillo@unicampania.it
}

Plants are currently experiencing increasing salinity problems due to irrigation with brackish water. Moreover, in fields, roots can grow in soils which show spatial variation in water content and salt concentration, also because of the type of irrigation. Salinity impairs crop growth and productivity by inhibiting many physiological and metabolic processes, in particular nitrate uptake, translocation, and assimilation. Salinity determines an increase of sap osmolality from about $305 \mathrm{mOsmol} \mathrm{kg} \mathrm{in}^{-1}$ control roots to about $530 \mathrm{mOsmol} \mathrm{kg}{ }^{-1}$ in roots under salinity. Root cells adapt to salinity by sequestering sodium in the vacuole, as a cheap osmoticum, and showing a rearrangement of few nitrogen-containing metabolites and sucrose in the cytosol, both for osmotic adjustment and oxidative stress protection, thus providing plant viability even at low nitrate levels. Several studies have already been carried out on durum wheat under salinity; most of them were performed on leaves. Only few data concern the effects of salinity on root metabolic profile, and how metabolite changes are related to the physiology of cells and root tissues.

Since it is unquestionable that the elucidation of fundamental molecular and physiological responses to salinity is instrumental to improving crops salt tolerance, in the present study uniform and non-uniform salinity have been simulated with a split-root system in which the root system was divided into two equal portions and each portion irrigated with $0 \mathrm{mM}$ (control) or $100 \mathrm{mM} \mathrm{NaCl}$ (salt stress) solution and $10 \mathrm{mM} \mathrm{KNO}_{3}$. Moreover, for the uniform salinity treatment (with the entire root system grown at 0 or $100 \mathrm{mM} \mathrm{NaCl})$, low and high nitrate concentrations $\left(0.1\right.$ and $10 \mathrm{mM} \mathrm{KNO}_{3}$, respectively) are applied. These conditions are used to study physiological root responses to salinity focusing on: (i) root ions accumulation and effect on some physiological parameters; (ii) osmolytes accumulation and contribution with ions to the osmotic balance of the root cells; (iii) expression and activity of the main enzymes involved in the synthesis of nitrogen-containing osmolytes; (iv) antioxidant response.

Durum wheat roots under salinity showed few changes in selected metabolites which allowed the plant viability even at low nitrate. This metabolic rearrangement was necessary to meet the demand for anti-stress agents including compatible solutes and antioxidants. Thus, while the sodium was used as osmoticum in the vacuole, mainly glycine betaine, sucrose, nystose, and 1fructofuranosylnystose at low nitrate, and glycine betaine, asparagine and proline at high nitrate were responsible for the osmotic adjustment, the assimilation of the excess of ammonium and the scavenging of ROS under salinity in the cytosol. The strong increase of the sole asparagine and glutamine in high nitrate split roots, either in control and salt stress conditions, suggests that the stressinduced adjustment is not a regional effect. On the contrary, the plant operates as an integrated system in which metabolic stress-induced signals spread in the plant and change the metabolism even in areas in which the stress conditions are not present. Notwithstanding this, different parts of plant root systems may behave as physiologically autonomous units, differing their responses to environmental signals, and preserving their own capability to supply the shoots with water, nutrients or assimilates. In this way one part of the root system can compensate the plant for a decreased supply or a loss of functionality by the other part, optimizing plant viability under heterogeneous water, nutrients or stressing conditions. 